\title{
ANÁLISIS DE COMPONENTES PRINCIPALES SOBRE DATOS MULTIESPECTRALES «LANDSAT-TM»E INTERPRETACIÓN DE CUBIERTAS VEGETALES EN LAS SIERRAS DE TEJEDA Y ALMIJARA
}

\author{
José A. CARREIRA y F.Xavier NIELL
}

RESUMEN. Análisis de componentes principales sobre datos multiespectrales "Landsat-TM» $e$ interpretación de cubiertas vegetales en las Sierras de Tejeda y Almijara. La consideración simultánea de todas las bandas de reflectancia en el análisis de imágenes multiespectrales teledetectadas, representa un incremento en el volumen de datos a procesar proporcionalmente mucho mayor que la ganancia real de información que se consigue, lo que se debe a la elevada correlación existente entre aquéllas. En este trabajo se aplica un análisis de componentes principales a datos multiespectrales Landsat TM de las Sierras de Tejeda y Almijara, con el objeto de sumarizar los mismos sin una pérdida significativa de información. Los dos primeros componentes principales obtenidos recogieron más del $99 \%$ de la varianza total original.

La imagen que se obtuvo a partir del primer componente principal representaba, aproximadamente, una suma ponderada de todas las intensidades de reflectancia en las distintas bandas consideradas (análoga, por tanto, a una fotografía en blanco y negro con una extensión en el infrarrojo) y mostró una interpretabilidad óptima para aspectos de topografía-relieve, red de drenaje y tipos litológicos. La imagen resultante de segundo componente principal (ya sin efecto de relieve) mostró la intensidad de señal "verde" del dosel vegetal y se correlacionaba significativamente con las obtenidas de la aplicación de índices espectrales de vegetación (RVI, NDVI) a los datos originales. El análisis preliminar de las imagenes indica problemas de deforestación grave en la zona (casi un 30\% de la superficie con suelos desnudos, y hasta un $85 \%$ si se incluyen areas con vegetación dispersa).

El análisis multivariado se ha confirmado como una herramienta útil para la interpretación de imágenes teledetectadas de la zona de estudio. Eventualmente, la obtención de la transformación de componentes principales por medio de un muestreo selectivo, proveería de algoritmos de transformación para los datos multiespectrales con los que se podría, por ejemplo, hacer un seguimiento de cambios en procesos tales como la deforestación.

Palabras clave. Andalucía, España, teledetección, A.C.P. (Análisis de Componentes Principales), cubiertas vegetales, Landsat-TM.

ABSTRACT. Principal component analysis of "Landsat-TM" multiespectral data and vegetation cover in the Tejeda and almijara mountain range. The simultaneous consideration of all reflectance bands during image processing of multiespectral, remotely-sensed data, usually involves a very large volume of data to handle. However, this increase in data volume is not followed by a similar increment in the volume of additional information that is accounted for, which is due to the existence of a very high correlation between reflectance bands. In this paper we report the results of applying a multivariate statistical tool (Principal Component Analysis, PCA) to the first four reflectance bands of a LANDSAT «Thematic Mapper» image of Sierra de Tejeda and Sierra de Almijara (Southern Spain). The aim of this analysis was to achieve a 
reduction in the volume of data to handle during image processing while avoiding a significant loss of information. The first two principal components resulting from the analysis accounted for more than $99 \%$ of the total variance in the original data set.

The image obtained through the first principal component transform could be interpreted as a weighed-sum image of all reflectance bands (thus similar to a black and white panchromatic photograph with an extension into the infrared wavelength). This image showed optimum feasibility for the interpretation of geological, hydrological and topographic features of the study area. The second principal component transform was able to extract the "green" signal of the vegetation canopy from the complex set of multiespectral data. Relief features (i.e., areas of shadow in north-facing slopes versus well-illuminated, south-facing slopes) did not appear in the resulting image (these features had already been accounted for by the first principal component), which is an advantage when studying vegetation cover in areas with steep relief. A strong possitive correlation was found between the second PCA image and those resulting from the application of standard spectral vegetation indexes (RVI and NDVI). The initial assesment of the images has shown that severe deaforestation is undergoing in the area. About $30 \%$ of the study area was occupied by bare soils. This figure went up to $85 \%$ if areas with scattered vegetation were also included.

Multivariate analysis has confirmed as a valuable tool for the interpretation of multiespectral satellite data from the study area. Eventually, the obtention of a principal component transform based on the selective sampling of appropiate subsets of pixels from the area would provide of algorithms that applied to the entire set in multitemporal images could, for instance, be used to monitor long-term and large-scale deaforestation processes in the region.

Key words. Andalusia, Spain, Landsat-TM, Principal Component Analysis (PCA), Remote sensing, Vegetation cover.

\section{INTRODUCCIÓN}

En los últimos años, y de forma cada vez más generalizada, se advierte la incorporación rutinaria de los potenciales que ofrece la teledetección espacial en estudios de la vegetación y evaluación del territorio. El volumen de datos acumulados desde el lanzamiento a partir de 1972 de la serie de satélites de observación terrestre "Landsat»es enorme, contándose en la actualidad con una serie temporal de 20 años. Esta gran cantidad de información no sólo es consecuencia de la progresiva extensión del periodo operativo de los satélites, sino que se relaciona también con la mejora que, paralelamente, se ha verificado en la resolución espectral y espacial de los sensores remotos que transportan (por ejemplo, para la serie Landsat, 7 bandas espectrales y una resolución espacial de $30 \mathrm{~m} * 30 \mathrm{~m}$ en los sensores Thematic Mapper; frente a sólo 4 bandas espectrales y una resolución espacial de $57 \mathrm{~m} * 79 \mathrm{~m}$ en los sensores MSS, sus predecesores).

Dicho tipo de mejoras posibilita, por ejemplo, una diferenciación más precisa entre distintas categorías de terreno mediante la selección de aquellas bandas de reflectancia en las que sus firmas espectrales se segregan con mayor claridad (Sabins 1987, Chuvieco 1990). Pero al mismo tiempo tales mejoras suponen, para una misma superficie de territorio, un incremento considerable en el número de datos a manejar que no se compensa en la misma proporción por la nueva información que se consigue. Ello se debe a la existencia de una cierta redundancia entre los valores de reflectancia en las distintas bandas espectrales TM (si bien menor que la existente entre bandas MSS), como se manifiesta al calcular la covarianza entre las mismas (Mulder 1974; véase tab. 1).

Para hacer frente a este problema, el Análisis de Componentes Principales (ACP) ha demostrado ser una herramienta de utilidad en el tratamiento de datos multiespectrales (e.g. Donker \& Muller 1977) puesto que permite eliminar el «ruido» y sumarizar la redundancia sin una pérdida sustancial de información. Asimismo, la discusión del significado de los 
componentes principales que absorben la mayor parte de la varianza facilita la interpretación conceptual de las imágenes resultantes de los mismos, que pueden llegar a representar, de hecho, imágenes temáticas.

Donker \& Muller (1977) aplicaron las técnicas de ACP en el procesado digital de imágenes Landsat MSS en un estudio sobre un área del Sureste de Holanda. Dicha zona se caracteriza por su escaso contraste topográfico y gran uniformidad general en cuanto a los tipos de unidades de terreno y vegetación existentes, lo que restringía la posibilidad de valorar toda la potencialidad de diferenciación que permite el ACP. En el presente trabajo, se llevó a cabo un procesado similar sobre datos Landsat TM pero correspondientes a un área mucho más diversificada en términos de relieve, litología y cubierta vegetal (Axarquía, y Sierras de Tejeda y Almijara entre las provincias de Málaga y Granada). Las imágenes derivadas del ACP, y su interpretación, se compararon con las obtenidas a partir de la aplicación de índices de vegetación «standard» como son el RVI o NDVI ( RRatio Vegetation Index», «Normalized Difference Vegetation Index»; Colwell 1974, Tucker 1979), imágenes en color falso e información contenida en cartografía temática disponible sobre el área de estudio (Elorza et al. 1979, Nieto 1987). Con ello se pretendía determinar la fiabilidad y aplicabilidad del análisis multivariado sobre imágenes digitales multiespectrales de la zona para eventuales estudios sobre problemas de deforestación en el área y/o de clasificación territorial.

\section{MATERIAL Y MÉTODOS}

\section{Área de estudio}

Las Sierras de Tejeda y Almijara (MálagaGranada) constituyen una alineación montañosa de relieve escarpado que se sitúa en la frontera del área de influencia subárida del
S.E. peninsular. En su amplio gradiente altitudinal (0-2065 m snm) están presentes variados tipos de vegetación (Nieto 1987) con diferentes grados de cobertura y desarrollo. En contraste, las grandes unidades tectónicas y litológicas presentes en el área son internamente uniformes, y se separan entre sí a través de límites netos y contínuos (Elorza et al. 1979). Asimismo, los suelos presentes en cada una de dichas unidades muestran un cierto grado de uniformidad. Tales características hacen del entorno de las Sierras de Tejeda y Almijara una zona idónea para este estudio en el que el ènfasis se pone en la diferenciación de áreas con distintos grados de desarrollo de la vegetación, en tanto que para otros rasgos fisiográficos sólo se pretende la posibilidad de su interpretabilidad general a gran escala.

Toda la zona es altamente suceptible frente procesos de desertificación, tanto por la referida influencia subárida, las elevadas pendientes de su relieve y el caracter arenoso y pobre de sus suelos; como por la elevada frecuencia y magnitud de los incendios forestales experimentados en las últimas décadas. De hecho, lo que hace apenas algo más de 3 décadas constituía una importante masa forestal de Pinus pinaster en la Sierra de Almijara (Ortuño \& Ceballos 1977) ha quedado reducido en la actualidad a pequeñas masas de carácter testimonial. Dicha circunstancia añade peso a la elección de las Sierras de Tejeda y Almijara como área de estudio, ya que podrían representar un caso paradigmático respecto a la verificación de procesos de degradación de la vegetación y pérdida de suelo en un intervalo de tiempo relativamente corto.

\section{Características y Procesamiento Digital de las Imágenes}

Para el presente estudio se emplearon las bandas espectrales TM1 (azul, 0.45-0.52 $\mu \mathrm{m}$ ),

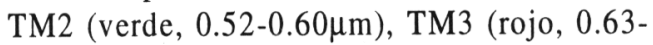
$0.69 \mu \mathrm{m})$ y TM4 (IRcercano, 0.76-0.90 $\mu \mathrm{m}$ ) de un sector de una escena Landsat «Thematic 
Mapper», libre de nubes, fechada en 15-061984 (coordenadas: N 37-29, W 4-42; path: 201 , row:34), con un total de $5 * 10^{5}$ píxeles de $30 \mathrm{~m} * 30 \mathrm{~m}$ sobre el terreno por cada banda. El procesado de las imágenes se realizó empleando un sistema ILWIS en el laboratorio de Procesamiento Digital de Imágenes del International Institute for Aerospace Survey and Earth Science (ITC, Holanda). Las imágenes fueron sometidas a una corrección automática para el « $6^{\text {th }}$ line stripping» $y$ dispersión atmosférica. La mejora del contraste de la imágenes se llevó a cabo por el procedimiento de realce linear.

Sobre los datos de las cuatro bandas originales, se calculó la matriz de varianzacovarianza y se aplicó un Análisis de Componentes Principales basado en una muestra completa de la imagen. En algunas ocasiones, se recomienda hacer el ACP sobre una muestra seleccionada de la imagen de la que se conoce que tiene una máxima variabilidad para los aspectos que pretenden estudiarse (Mulder 1974). De esta forma se puede optimizar la transformación del ACP a las preferencias del usuario para, por ejemplo, separar más eficientemente determinadas categorías de terreno que, aunque no representan una fracción importante de la varianza total de la imagen (y, por tanto, quedarían mal separadas tras una transformación de ACP basada en una muestra completa de la misma), interesa destacar de acuerdo con los objetivos específicos del estudio. En nuestro caso, no fue necesario un muestreo selectivo ya que la varianza entre distintas categorías de terreno estaba bien distribuida por toda la imagen.

Las imágenes derivadas de la transformación por componentes principales se compararon con las obtenidas a través de métodos rutinarios habitualmente empleados en el procesamiento digital de imágenes para labores de fotointerpretación general. Se obtuvieron las siguientes imágenes:
- Elaboración de una imagen compuesta en color falso, con las bandas TM2, TM3 y TM4. Previamente, las imágenes originales de cada banda individual habían sido sometidas a un realce lineal de contraste. Asimismo, para evitar en lo posible un efecto exagerado de sombras debido al relieve abrupto de la zona, y obtener colores espectrales más puros en la imagen compuesta, a cada banda se le calculó su coseno direccional según las fórmulas: cos $\stackrel{\mathrm{a}}{=}=\mathrm{I}_{\mathrm{TM} 2} / \mathrm{R}, \cos \beta=\mathrm{I}_{\mathrm{TM} 3} / \mathrm{R}, \cos \partial=\mathrm{I}_{\mathrm{TM} 4} / \mathrm{R}$; donde $\mathrm{I}_{\mathrm{TMX}}$ : valor en cada píxel de la intensidad de reflectancia en la banda $\mathrm{x} ; \mathrm{y} \mathrm{R}=$ $\sqrt{ }\left(\mathrm{I}_{\mathrm{TM} 2}{ }^{2}+\mathrm{I}_{\mathrm{TM} 3}{ }^{2}+\mathrm{I}_{\mathrm{TM} 4}{ }^{2}\right)$.

- Elaboración de imágenes mediante la aplicación de índices de vegetación. Se han desarrollado numerosos índices espectrales para caracterizar las cubiertas vegetales en imágenes teledetectadas. Los más comunes emplean los valores de reflectancia en el IRcercano, y en el rojo (absorción de la clorofila), combinados en forma de proporción (Tucker 1979). Tales índices se correlacionan bien con diversos parámetros de la vegetación como son: biomasa, índice foliar, productividad, \% cobertura de material verde y actividad fotosintética (Colwell 1974, Asrar 1984, Sellers 1985, entre otros). En este trabajo se emplearon dos de los más sencillos de tales índices, el RVI (Ratio Vegetation Index) y NDVI (Normalized Difference Vegetation Index), donde: $\mathrm{RVI}=\left(\mathrm{I}_{\mathrm{TM} 4} / \mathrm{I}_{\mathrm{TM} 3}\right)$, y NDVI $=\left(\mathrm{I}_{\mathrm{TM} 4}-\mathrm{I}_{\mathrm{TM} 3}\right) /$ $\left(\mathrm{I}_{\mathrm{TM} 4}+\mathrm{I}_{\mathrm{TM} 3}\right)$.

\section{RESULTADOS Y DISCUSIÓN}

\section{Resultados del ACP}

En la tabla 1 se muestran la varianza y covarianza entre los valores de reflectancia en las bandas TM1, TM2, TM3 y TM4. Cabe destacar la escasa varianza de los datos correspondientes a la banda TM2 (verde, $11.5 \%$ de la varianza total) en relación a los de la banda TM4 (infrarrojo cercano, $41.3 \%$ de la 
varianza total). La redundancia entre bandas (correlación entre los valores de reflectancia en las distintas bandas para cada píxel de terreno) era elevada, como se deduce de los valores altos de covarianza, sobre todo entre la banda TM4 con las bandas TM1 y TM3.

En la tabla 2 se muestran los valores y vectores propios resultantes del ACP. La varianza medida a lo largo del primer componente principal (primer valor propio) fue 371.3 , la que corresponde al segundo fue 31.8. Así pues, juntos, el $1^{\circ}$ y $2^{\circ}$ componentes principales recogían más del $99 \%$ de la varianza total original (407.1). Ello representa una considerable compresión de los datos, desde las 4 bandas originales empleadas para el análisis $\left(2 * 10^{6}\right.$ datos $)$ a sólo dos componentes principales $\left(10^{6}\right.$ datos $)$ con una pérdida de información inferior al $1 \%$.

Huete \& Jackson (1987), con datos multiespectrales de una serie de doseles vegetales creados experimentalmente con diferentes suelos, tipos de hojarasca y biomasa; obtuvieron también una reducción a un modelo de sólo dos componentes principales que recogían más del $99 \%$ de la varianza. Donker \& Muller (1976) con datos Landsat MSS del sur de Holanda, en áreas relativamente homogéneas, explican el $98 \%$ con los dos primeros componentes principales. No obstante, a partir de datos multiespectrales obtenidos sobre terrenos reales, mas heterogéneos, no suele alcanzarse una compresión de los datos tan espectacular (normalmente se requieren los tres primeros componentes principales para no perder una cantidad significativa de información).

El resultado de proyectar el conjunto de píxeles de nuestras imágenes originales sobre el nuevo juego de ejes ortogonales son 4 nuevas imágenes correspondientes a cada uno de los componentes principales.

\section{Interpretación de la imagen del PC1}

En la figura 1a se muestra el «espectro» de los dos primeros vectores propios, esto es, se representan sus factores de carga frente a la longitud de onda media de las correspondientes bandas (véase también la tab. 2). Los coeficientes de carga indican el modo en que las 4 bandas TM originales son transformadas en el $1^{\circ}, 2^{\circ}, 3^{\circ}$ y $4^{\circ}$ ejes principales (en adelante, PC1, PC2, PC3 y PC4 respectivamente). Como se observa en la tabla 2 y figura 1 , todos los

\begin{tabular}{lcccc}
\hline Banda: & $\begin{array}{c}\text { TM1 } \\
\text { (azul) }\end{array}$ & $\begin{array}{c}\text { TM2 } \\
\text { (verde) }\end{array}$ & $\begin{array}{c}\text { TM3 } \\
\text { (rojo) }\end{array}$ & $\begin{array}{c}\text { TM4 } \\
\text { (IRcercano) }\end{array}$ \\
\cline { 2 - 5 } TM1 $(0.45-0.52 \mu \mathrm{m})$ & $97.9(24.1)$ & & \\
TM2 $(0.52-0.60 \mu \mathrm{m})$ & 65.7 & $46.9(11.5)$ & \\
TM3 $(0.63-0.69 \mu \mathrm{m})$ & 92.0 & 65.2 & $92.7(22.8)$ & \\
TM4 $(0.76-0.90 \mu \mathrm{m})$ & 104.3 & 76.79 & 105.3 & $169.5(41.3)$ \\
& \multicolumn{5}{c}{ Varianza total: $\sum$ diagonal $=407.1$} \\
\hline
\end{tabular}

Tabla 1. Matriz de Varianza-Covarianza de los valores de intensidad de reflectancia en las bandas TM1 a TM4 para el conjunto de píxeles de una imagen Landsat «TM» de las Sierras de Tejeda y Almijara, MálagaGranada (los valores entre paréntesis representan el porcentaje de la varianza total que corresponde a cada banda). Variance-covariance matrix of reflectance intensity data for bands TM1 to TM4 of a Landsat "TM» image of Sierras de Tejeda and Almijara, Málaga-Granada, Spain (data shown in parenthesis are total variance for each band). 

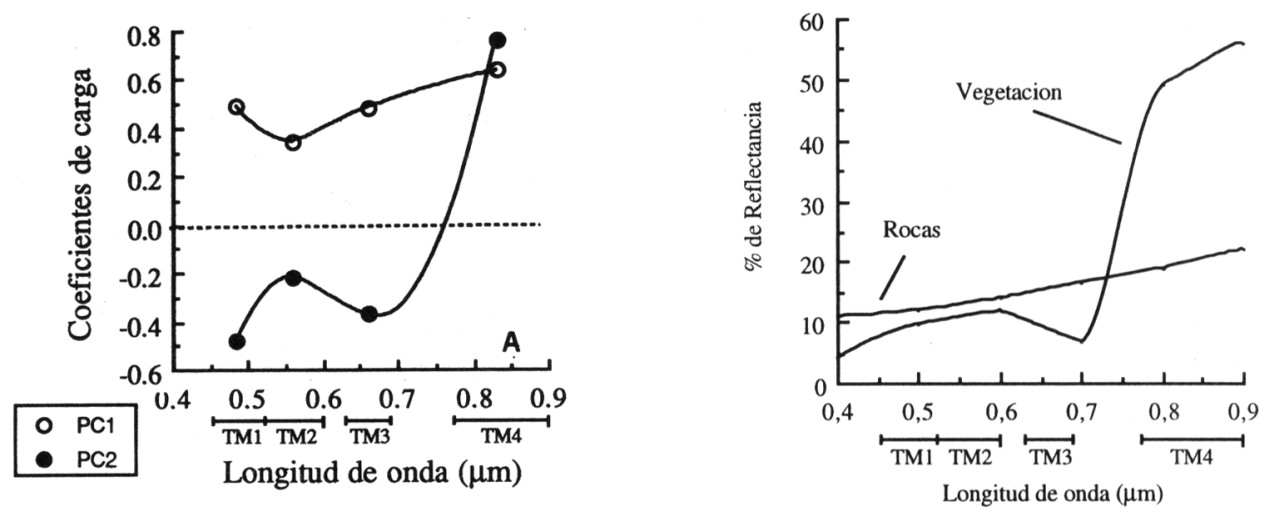

Figural.- A) Relación entre los valores de los coeficientes de carga en el PC1 y PC2, y la longitud de onda media de las bandas espectrales TM a que corresponden; o "espectros" de los vectores propios. B) Firmas espectrales típicas de terrenos ocupados por vegetación vigorosa, o rocas y suelo desnudo (\% de la radiación incidente que es reflejado en cada longitud de onda por dichas unidades de terreno). Adaptado de Sabins (1989). A) Relationship between the mean wavelength corresponding to each spectral band and their respective load coefficients in the first and second principal components (or "spectrum» of the eigen vectors). B) Typical spectral signature of terrain units covered by dense vegetation stands, or rocks and bare soils (\% of reflectance typically shown by each of those terrain units in each spectral band). Adapted from Sabins (1989).

factores de carga de las cuatro bandas TM en el PC1 son positivos y de magnitud similar, con valores que oscilan alrededor de 0.5 aunque aumentando ligeramente para valores crecientes de longitud de onda. Cada píxel de la nueva imagen se ha obtenido mediante la transformación: $\mathrm{IPC}_{1}=0.489 * \mathrm{ITM}_{1}+$ $0.347 * \mathrm{ITM}_{2}++0.484 * \mathrm{ITM}_{3}+0.637 * \mathrm{ITM}_{4}$.

Ello sugiere que la imagen resultante de la transformación del PC1 representa una imagen promedio del conjunto de intensidades de reflectancia en las bandas del azul, verde, rojo e infrarrojo cercano; por lo que podría interpretarse como análoga a una fotografía en blanco y negro con una extensión en el infrarrojo.

Nótese lo significativo de la similitud entre la forma del «espectro» del PCl (fig. 1a) y la forma característica de las firmas espectrales que presentan terrenos con suelos desnudos o rocas (fig. 1b), materiales que, típicamente, tienen índices de reflectancia que se elevan progresiva pero suavemente con el aumento de la longitud de onda de la radiación incidente (Sabins 1989). Así, la imagen derivada del PC1 (no se muestra) ofrecía una buena interpretabilidad general de la zona, poniéndose claramente de manifiesto diversos rasgos fisiográficos como son relieve y red de drenaje, lineamientos, fracturas y otras características tectónicas del área. Los grandes patrones de drenaje observables en la imagen del PC1, se correspondían fielmente con el trazo de los principales rios y arroyos que se muestran en la figura 2.

Asimismo, en la imagen derivada del PC1 fueron fácilmente reconocibles los grandes tipos litológicos presentes en el área, con gran fidelidad respecto a la información contenida en el mapa geológico de la zona (Elorza et al. 1979; véase también fig. 2). Destacaba particularmente la nitidez del contacto entre los mármoles dolomíticos triásicos claros de las Unidades de Sierra Tejeda y las Alberquillas, y los materiales paleozóicos subyacentes, más oscuros, del Manto de los 


\begin{tabular}{lccrr}
\hline & \multicolumn{4}{c}{ Vectores propios } \\
\hline Banda & PC1 & PC2 & PC3 & \multicolumn{1}{c}{ PC4 } \\
TM1 $(0.45-0.52 \mu \mathrm{m})$ & 0.489 & -0.479 & 0.725 & 0.072 \\
TM2 $(0.52-0.60 \mu \mathrm{m})$ & 0.347 & -0.216 & -0.291 & 0.865 \\
TM3 $(0.63-0.69 \mu \mathrm{m})$ & 0.484 & -0.371 & -0.619 & -0.495 \\
TM4 $(0.76-0.90 \mu \mathrm{m})$ & 0.637 & 0.766 & 0.072 & -0.041 \\
\cline { 2 - 5 } Valores propios & 371.3 & 31.83 & 3.30 & 0.66 \\
\% variancia total recogida & & & & \\
por cada valor propio & 91.20 & 7.82 & 0.81 & 0.16 \\
\% acumulado & 91.20 & 99.02 & 99.83 & 100 \\
\hline
\end{tabular}

Tabla 2. Valores propios y matriz de vectores propios resultante del análisis de componentes principales sobre una imagen digital Landsat «TM» de las Sierras de Tejeda y Almijara, Málaga-Granada. Eigen values and matrix of eigen vectors resulting from the application of a Principal Component Analysis to a Landsat "TM» image of Sierra de Tejeda and Almijara, Málaga-Granada, Spain.

Guajares (gneises y esquistos). Igualmente, dentro de la Unidad de Sierra Tejeda, en su flanco Sur, fue fácil la diferenciación entre los afloramientos de esquistos verdes paleozóicos y los mármoles triásicos que constituyen el núcleo de la misma. La Sierra de Almijara aparecía en la imagen del PC1 como un conjunto bien individualizado, a modo de cuña con vértice orientado al Oeste que cabalga sobre el Manto de los Guajares, apreciándose la intensa fracturación que ha dado lugar al relieve abrupto que la caracteriza. De la interpretación de la imagen PC1, aunque con mayor dificultad, pudo seguirse el contacto entre la Unidad de las Alberquillas al Sur, y la de la Herradura al Norte, dentro de la Sierra de Almijara.

\section{Interpretación de la imagen del PC2, e índices de vegetación}

En relación a la interpretación del PC2, en la tabla 2 y figura 1 a se observa que las bandas TM1(azul), TM2 (verde) y TM3 (rojo) tienen coeficientes de carga de signo contrario al de la banda TM4 (infrarrojo cercano). Esta banda, TM4, tiene un componente de carga positivo y muy elevado en el PC2 (0.77). La imagen del $\mathrm{PC} 2$ se construye según la transformación: $\mathrm{IPC}_{2}=-0.479 * \mathrm{ITM}_{1}$ $0.216 * \mathrm{ITM}_{2}-+0.371 * \mathrm{ITM}_{3}+0.766 * \mathrm{ITM}_{4}$.

La forma del «espectro» del PC2 recuerda la de la firma espectral típica de la vegetación (fig. 1a y 1 b, respectivamente), en particular por el brusco incremento entre el rojo y el IRcercano, así como también por el pico en el verde y la baja respuesta en el rojo. El componente «verde» del dosel vegetal tiene la propiedad de reflejar una considerable proporción de la radiación que recibe en la banda del infrarrojo (y, en menor medida, también en el verde), en tanto que absorbe en el rojo (Sabins 1989). Por tanto, las porciones del área de estudio ocupadas por vegetación bien desarrollada tendrán valores relativamente elevados de reflectancia en la banda TM4 (que contribuye fuertemente al PC2, con un coeficiente de carga positivo y alto), y reflectancias relativamente bajas en las bandas TM3 (que contribuyen con coeficientes de carga muy negativos al PC2).

Por tanto, el PC2 resultó ser una transformación capaz de extraer la señal "verde" de la vegetación de entre un conjunto complejo de datos multiespectrales, y su imagen indicaba distintos grados de desarrollo y 
cobertura del dosel vegetal. Esta interpretación se confirmó al comprobar la elevada correlación entre la imagen obtenida por el PC2 y las que se obtuvieron de aplicar índices de vegetación como el RVI y NDVI $(0.82$ y 0.89 respectivamente, $\mathrm{p}<0.01)$. Huete \& Jackson (1987), utilizando datos multiespectrales de distintos tipos de coberturas simuladas experimentalmente, comprobaron que el ACP podía mejorar significativamente la extracción de la señal de la vegetación en comparación con diversos índices de vegetación.

En la imagen resultante de la transformación por el PC2 (la imagen no se muestra) no aparecieron ya efectos de sombrarelieve, y el tipo de rasgos comentados para la imagen del PC1 eran ahora irreconocibles. En su lugar, aparecía una gradación desde zonas con gran desarrollo de la vegetación en tonos muy claros (e.g. bosques densos y vegetación de ribera a lo largo de los arroyos), hasta zonas con suelo desnudo en grises muy oscuros (e.g. cumbres de la Sierra de Tejeda, o áreas recientemente incendiadas en la Sierra de Almijara).

El patrón de los principales cursos fluviales de la zona (Vélez, Algarrobo, Torrox..., fig. 2) fue fácilmente reconocible por los tonos muy claros que presentaban sus vegetaciones de ribera en la imagen PC2. Asimismo, destacaban en tonos claros los matorrales densos y repoblaciones de pinar asociadas a la solana de la Sierra de Tejeda sobre los materiales esquistosos. Las zonas desprovistas de vegetación en torno a las cumbres más elevadas (Maroma 2065m, Lucero $1771 \mathrm{~m}$, Malas Camas 1824...) aparecían en tonos muy oscuros. En la Sierra de Almijara, en las cercanías de las poblaciones de Cómpeta y Frigiliana, se apreciaban manchas negras muy marcadas que corresponderían a áreas recientemente incendiadas. Asimismo, los pinares densos de El Alcazar, en el término de Alcaucín, eran fácilmente reconocibles como una mancha redondeada de tonos claros en la parte superior izquierda de la imagen PC2. Más hacia el Este, aparecía otra mancha de colores claros correspondiendo a las masas de robledales y encinares en la vertiente granadina de la Sierra de Tejeda.

Estos mismos patrones generales de distribución de manchas de vegetación podían apreciarse en la imagen de color falso (no se muestra). En este caso, las áreas predominantemente ocupadas por vegetación bien desarrollada aparecen en tonos rojizos de más o menos intensidad. Una primera impresión que se deriva de dicha imagen compuesta de color falso y de la del PC2 es la elevada proporción de terrenos desforestados en la zona de estudio. Analizando los rangos de valores obtenidos tras la aplicación del índice de vegetación NDVI (fig. 2, arriba) y haciendo una asignación aproximada de los tipos de cobertura de terreno a que corresponden, se confirma la apreciacion anterior (tab. 3; los tipos de cobertura de terreno se identificaron mediante un muestreo selectivo, en las imagenes de los índices de vegetación, de píxeles que tuvieran valores dentro de los intervalos seleccionados, y su posterior localización geográfica y comprobación en el campo de la vegetación existente).

Debido a que se carece por el momento de datos concretos para las Sierras de Tejeda y Almijara, hemos efectuado una revisión bibliográfica sobre relaciones entre valores de NDVI y cobertura, biomasa e índice foliar en zonas con vegetación de características similares (Ringrose \& Matheson 1987, Elvidge \& Lyon 1985, Huete et al. 1985, Huete \& Jackson 1987, Avery \& Berlin 1985). Las rocas y suelos desnudos tienen, característicamente, valores de NDVI que oscilan en torno a cero. Valores de NDVI entre 0.1 y 0.2 se corresponden aproximadamente con terrenos ocupados por vegetación dispersa, con una cobertura total que oscila alrededor del $40 \%$, rara vez superando el $60 \%$; y valores de índice foliar en torno a 0.5 . Valores de NDVI cercanos 

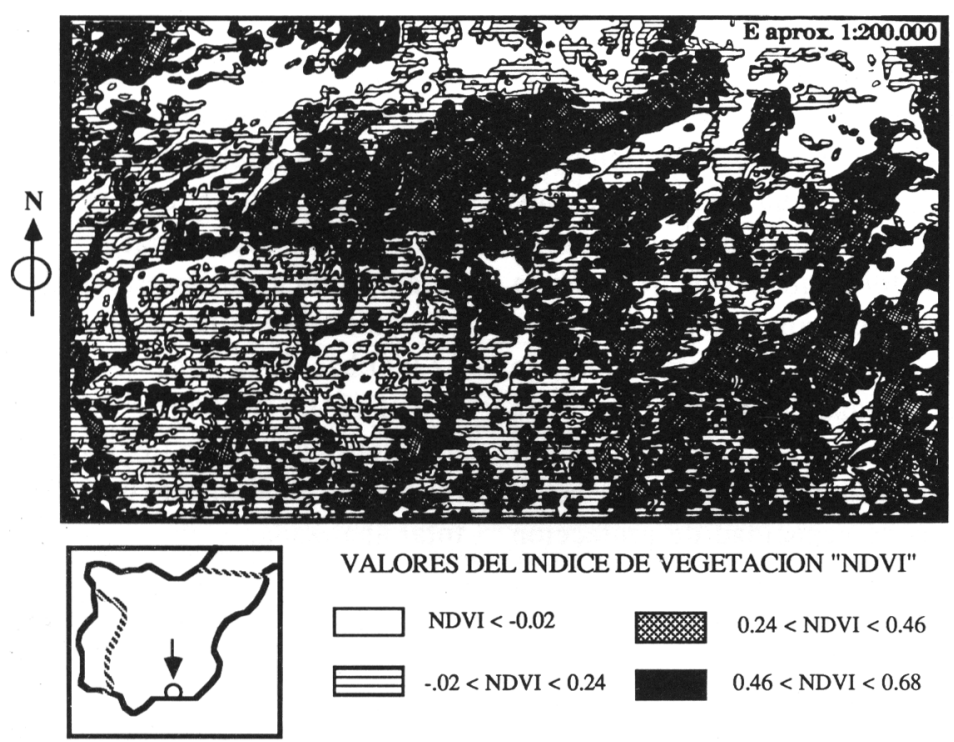

VALORES DEL INDICE DE VEGETACION "NDVI"

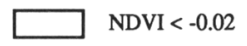

$-.02<\mathrm{NDVI}<0.24$

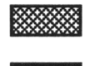

$0.24<\mathrm{NDVI}<0.46$

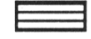

(1)

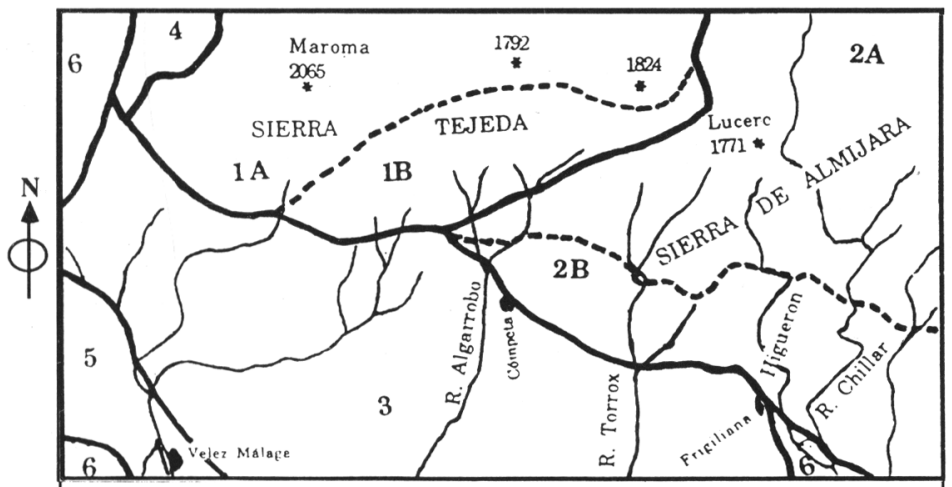

UNIDADES LITOLOGICAS

$$
\begin{gathered}
\text { Complejo } \\
\text { Alpujárride }
\end{gathered}\left[\begin{array}{l}
\text { Unidad de Sierra Tejeda } \\
\begin{array}{l}
\text { Triásico, mármoles dolomíticos } \\
\text { muy kakiritizados, con biotitas }
\end{array} \\
\begin{array}{l}
\text { 3: Manto de Guajares: Paleozóico, esquistos y geneises } \\
\text { 4: Unidad de Salares: Triásico, mármoles con biotita }
\end{array}
\end{array}\right.
$$

5: Complejo Maláguide.Paleozoico

6: Postorogénico. Né́geno y Cuaternario

Figura 2.- Situación geográfica y características generales del área de estudio. En la figura superior se representan con diferentes tramas las áreas con valores similares del índice de vegetación NDVI (véase tab. 3 para su equivalencia con distintos tipos de categorías de terreno). El esquema de la parte inferior representa la interpretación sobre la imagen en color falso y del PCl de las principales líneas de drenaje, elementos del relieve y tipos litológicos, completada con información de los mapas topográfico y geológico de la zona. Geographic position and general characteristic of the study area. Above: map showing the distribution of values for the Normalized Difference Vegetation Index (see Table 3 for their equivalence in terms of vegetation cover type). Below: Photointerpretation based on the analysis of the false colour composite image and the first principal component image, showing the main drainage patterns, relief features and lithology of the area. Additional data were gathered from topographic and geologic maps of the region. 
a 0.3 se corresponden típicamente con una vegetación ya más cerrada, con hasta un $75 \%$ de cobertura e índice foliar entre 0.5 y 1 . Valores de NDVI entre 0.4 y 0.7 son típicos de terrenos ocupados por una vegetación muy densa y/o boscosa, con cobertura total entre el $90-100 \%$ e índice foliar que puede llegar hasta 2.5. Haciendo una extrapolación general de estas correspondencias a nuestra zona de estudio, se derivó que hasta el $85 \%$ de la superficie abarcada por las imágenes estaría ocupada por suelo desnudo o por una vegetación dispersa y con poca capacidad de protección contra la erosión (tab. 3).

Aunque tienen validez como indicadores de carácter general, estas consideraciones han de ser tomadas con cautela debido al grado de incertidumbre asociado a la extrapolación de relaciones entre valores de NDVI y variables de la vegetación obtenidas en lugares diferentes y con imágenes de fechas distintas a las que corresponden al estudio al que se quieren aplicar. Ademas, en el caso de zonas semiáridas, existe una dificultad añadida debido a la distorsión que la señal del suelo introduce en índices como el NDVI y RVI (Huete \& Jackson 1987). Una posible vía para evitar este inconveniente sería la obtención de factores de corrección mediante muestreos en el campo para la aplicación del índice SAVI (Soil Adjusted Vegetation Index, Huete 1988). Por otro lado, el NDVI es sensible básicamente a la señal "verde" de la vegetación. En la fecha en que fueron tomadas las imágenes empleadas en este estudio (Junio), una buena parte de la vegetación de tipo herbáceo, que puede jugar un papel muy relevante en la protección del suelo, estaría ya agostada; y los terrenos ocupados por este tipo de vegetación darían respuestas muy bajas a la aplicación de los índices de vegetación.

En todo caso, es muy indicativo que la frecuencia absoluta máxima de píxeles con un determinado valor de NDVI (31252 píxeles, 2813 has), se obtuvo para el valor de NDVI=0; $y$, por tanto, para terrenos con exactamente el mismo valor de reflectancia en el rojo y en el infrarojo. Píxeles con este valor e inferiores claramente representan zonas desprovistas de vegetación con tejidos verdes en cantidad suficiente como para influenciar las caracteristicas espectrales de la radiación reflejada. Este tipo de terrenos ocupa unas 12.000 has de la zona de estudio (tab. 3). En contraste, las áreas con vegetación boscosa 0 matorrales densos no llega a las 6000 ha, lo que representa escasamente un $13 \%$ de la superficie total analizada.

\section{Interpretación de las imágenes del PC3 y PC4}

Las imágenes correspondientes al PC3 y PC4 pudieron ser desechadas ser desechadas del análisis. No aportan ninguna información de valor, sino que únicamente muestran «ruido» de las imágenes originales. En el PC3 hay una contribución importante de la banda TM1 (azul) (tab. 2), lo que indicaría que dicho eje extrae el ruido de «scattering» atmosférico. El PC4 probablemente actúa a modo de filtro que extrae el defecto de «6th line stripping» que presentan los sensores Thematic Mapper, de ahí el patrón de líneas paralelas blancas y negras, sin ningún otro rasgo a destacar, que aparecía en la correspondiente imagen (no se muestra).

\section{CONCLUSIONES}

Con imágenes Landsat-TM de las Sierras de Tejeda y Almijara, el ACP se confirma como una herramienta útil para la compresión de datos multiespectrales sin pérdidas significativas de información. Además, la interpretación de los vectores propios permitió asignar a sus correspondientes imágenes determinados "significados" que resultaron útiles para su análisis. Así, la transformación 


\begin{tabular}{|c|c|c|c|c|c|c|}
\hline N.D.V.I. & R.V.I. & $\begin{array}{l}\text { Tipo aproximado } \\
\text { cubierta vegetal }\end{array}$ & $\begin{array}{c}\text { no de } \\
\text { píxeles }\end{array}$ & $\begin{array}{l}\text { superficie } \\
\text { (ha) }\end{array}$ & $\begin{array}{c}\% \text { superficie } \\
\text { total }\end{array}$ & $\begin{array}{l}\% \text { superficie } \\
\text { acumulado }\end{array}$ \\
\hline$-0.42<=x<-0.20$ & $0.03<=x<0.45$ & $1 \mathrm{a}, 2,3 \mathrm{a}, 4$. & 5263 & 473.7 & 1.05 & 1.05 \\
\hline$-0.20<=x<-0.02$ & $0.45<=x<1.03$ & $1 a-b, 3 a-b$ & 132913 & 11962.2 & 26.6 & 27.6 \\
\hline$-0.02<=x<0.24$ & $1.03<=x<2.01$ & $5,6,7$ & 297593 & 26783.4 & 59.5 & 87.2 \\
\hline $0.24<=x<0.46$ & $2.01<=x<3.71$ & 8,9 & 60929 & 5483.6 & 12.2 & 99.3 \\
\hline $0.46<=x<0.68$ & $3.71<=\mathrm{x}<8.23$ & $10,1112,13$ & 3302 & 297.2 & 0.66 & 100 \\
\hline
\end{tabular}

1a: Afloramientos de mármoles claros. 1b: Afloramientos de materiales paleozóicos oscuros. 2: Sombras.

3a y 3 b: Suelos desnudos sobre mármoles o materiales paleozóicos, respectivamente; pastizales agostados.

4: Areas recien incendiadas. 5: Tomillares y lastonares. 6: Matorrales abiertos.

7: Cultivos marginales y barbechos de la Axarquía. 8: Pinares abiertos y matorrales sobre mármoles.

9: Cultivos frutales (aguacates...) sobre materiales paleozóicos. 10:Formaciones densas de monte alto.

11: Vegetación de ribera. 12: Pinares densos y bosques de frondosas.

13: Cultivos intensivos (huertas...) sobre materiales paleozóicos y postorogénicos.

*: Sin corrección por pendientes.

Tabla 3. Intervalos de valores obtenidos tras la aplicación de los índices de vegetación N.D.V.I. ( Normalized Difference Vegetation Index») y R.V.I. («ratio vegetation Index») a los datos multiespectrales «TM» originales de una imagen Landsat de las Sierras de Tejeda y Almijara, tipos aproximados de cubierta vegetal a que corresponden, y número de píxeles y superficie de terreno que ocupan (la adscripción de los tipos de cubierta a cada intervalo de valores de índices de vegetación se realizó mediante el muestreo en las imágenes de píxeles con valores de NDVI característicos del intervalo, su localización geográfica, y la comprobación en el campo). Range of values obtained by the application of vegetation indexes (NDVI: Normalized Difference Vegetation Index; RVI: Ratio Vegetation Index) to multiespectral data of a Landsat "TM» image of Sierra de Tejeda and Almijara, corresponding vegetation cover types, and number of pixels and \% surface area occupied by each vegetation type (vegetation types were assigned to each range of vegetation index values by coupling a selective sampling of pixels from the image with a field survey).

obtenida para el PC1 representaba una suma ponderada de las intensidades de reflectancia en distintas bandas, y su imagen mostró una interpretabilidad óptima para rasgos fisiográficos generales del área. El PC2 extrajo la señal "verde" del dosel vegetal de entre el conjunto complejo de datos multiespectrales de reflectancia del terreno, lo que puede representar una buena alternativa a los índices de vegetación más comunmente utilizados en el análisis digital de imágenes teledetectadas.

El análisis tanto de la imagen del PC2 como de los rangos de valores obtenidos con la aplicación de los índices de vegetación NDVI y RVI a los datos multiespectrales de las Sierras de Tejeda y Almijara, indica una proporción muy importante de terreno con suelos desnudos (27\%) o con vegetación muy dispersa (59\%).
Ello verifica la gravedad de los procesos de deforestación que se han verificado en la zona en las últimas décadas. No obstante, esta interpretación preliminar de los datos deberá ser concretada mediante la caracterización en el campo de las firmas espectrales de los distintos tipos de vegetación presentes en el área.

En este estudio, la realización del ACP se basó en la muestra completa de todos los píxeles. El siguiente paso para la mejora del resultado obtenido pasaría por la obtención de la transformación de Componentes Principales pero basándose en datos procedentes de un muestreo selectivo, realizado exclusivamente sobre píxeles que contuvieran la máxima varianza entre sí en términos de su cobertura vegetal. Ello permitiría, en su caso, obtener una transformación específica para la zona que 
permitiera monitorizar de forma rápida y eficiente procesos tales como la deforestación y desertificación.

\section{BIBLIOGRAFÍA}

ASRAR G., M. FUCHS, E.T. KANEMASN \& J.L. HATFIELD -1984- Estimating absorbed photosynthetic radiation and leaf area index from spectral reflectance in wheat. Agronomy Journal, 76:300-306.

AVERY T.E. \& G.L. BERLIN -1985-Fundamentals of remote sensing and airphoto interpretation. MacMillan. 472 pp.

COLWELL, J.E. -1974-Vegetation canopy reflectance. Remote Sensing of the Environment, 3: 175-183.

CHUVIECO, E. -1990- Fundamentos de teledetección espacial. Ed. Rialp, Madrid. 455 pp.

DONKER N.H.W \& N.J. MULDER -1976- Analysis of MSS digital imagery with the aid of principal component transform. ISP Commission VII.

ELORZA J.J., V. GARCÍA-DUEÑAS, J. MATAS \& L. MARTÍN -1979- Hoja geológica de Zafarraya (18-43). Mapa Geológico de España, 1:50000. IGME.

ELVIDGE C.D. \& R.J.P. LYON -1985-Influence of rock-soil spectra variation on the assesment of green biomass. Remote Sensing of the Environment, 17: 265-279.

HUETE A.R. -1988- A soil-adjusted vegetation index. Remote Sensing of the Environment, 25: 295-309.

HUETE A.R., R.D. JACKSON \& D.F. POST -1985Spectral response of a plant canopy with different soil backgrounds. Remote Sensing of the Environment, 17: 37-53.

HUETE A.R. \& R.D. JACKSON -1987- Suitability of spectral indices for evaluating vegetation characteristics on arid rangelands. Remote Sensing of the Environment, 23:213-232.

MULDER N.J. -1974- Data compresion and data reduction techniques for the visual interpretation of multiespectral images. ITC-Journal, 1974-3.

NIETO J.M. -1987-Estudio fitocenológico de las Sierras de Tejeda y Almijara (MálagaGranada). Tesis doctoral, Universidad de Malaga. 557 pp.
ORTUÑO F. \& A. CEBALLOS -1977- Los bosques españoles. Ed. Incafo, Madrid. $251 \mathrm{pp}$.

RINGROSE S. \& W. MATHESON -1987-Spectral assesment of indicators of range degradation in the Botswana hardveld environment. Remote Sensing of the Environment, 23: 379-396.

SABINS F.F. -1987- Remote sensing: Principles and interpretation. Freeman, New York. 449 p.

SELLERS P.J. -1985- Canopy reflectance, photosynthesis and transpiration. Int. Journal of Remote Sensing, 6:1335-1372.

TUCKER C.J. -1979- Red and photographic infrared linear combinations for monitoring vegetation. Remote Sensing of the Environment, 8:127-150.

Aceptado para su publicación en Junio de 1995

Dirección de los autores. Departamento de Geología y Ecología. Universidad de Malaga. Campus de Teatinos s/n 29071-Malaga (España) 